International Journal of Modern Physics A

(C) World Scientific Publishing Company

\title{
EFFECTS OF FINAL STATE INTERACTIONS ON HADRONIC CHARMLESS $B$ DECAYS
}

\author{
Hai-Yang Cheng \\ Institute of Physics, Academia Sinica \\ Taipei, Taiwan 115, ROC
}

\begin{abstract}
Final-state rescattering effects on direct $C P$ violation in charmless hadronic $B$ decays
\end{abstract} and on the polarization anomaly in $B \rightarrow \phi K^{*}$ are discussed.

\section{Introduction}

Why should we study final-state interactions (FSIs) in charmless hadronic $B$ decays seriously and urgently ? It is largely to do with the recent observation of direct $C P$ violation. A first confirmed observation of direct $C P$ partial rate asymmetry in charmless $B$ decays was established recently in $\bar{B}^{0} \rightarrow K^{-} \pi^{+}$by BaBar ${ }^{1]}$ and Belle. 2 The combined BaBar and Belle measurements of $\bar{B}^{0} \rightarrow \rho^{ \pm} \pi^{\mp}$ imply a $3.6 \sigma$ direct $C P$ asymmetry in the $\rho^{+} \pi^{-}$mode ${ }^{3}$ As for direct $C P$ violation in $B^{0} \rightarrow \pi^{+} \pi^{-}$, a $5.2 \sigma$ effect was claimed by Belle, $\frac{4}{4}$ but it has not been confirmed by BaBar $\underline{5}$

Table 1. Comparison of $\mathrm{pQCD}$ and QCD factorization (QCDF) predictions of direct $C P$ asymmetries (in \%) with experiment. Also shown in the last column are the FSI modifications to QCDF predictions taken from [6].

\begin{tabular}{lrrrr}
\hline Modes & Expt. & pQCD & QCDF & QCDF+FSI \\
\hline $\bar{B}^{0} \rightarrow K^{-} \pi^{+}$ & $-11 \pm 2$ & $-17 \pm 5$ & $4.5_{-9.9}^{+9.1}$ & $-14_{-3}^{+1}$ \\
$\bar{B}^{0} \rightarrow \rho^{+} \pi^{-}$ & $-48_{-15}^{+14}$ & $-7.1_{-0.2}^{+0.1}$ & $0.6_{-11.8}^{+11.6}$ & $-43 \pm 11$ \\
$\bar{B}^{0} \rightarrow \pi^{+} \pi^{-}$ & $37 \pm 24$ & $23 \pm 7$ & $-6.5_{-13.3}^{+13.7}$ & $64_{-8}^{+3}$ \\
\hline
\end{tabular}

Table 1 shows comparison of the model predictions of direct $C P$ asymmetries with the world averages of experimental results. ${ }^{3}$ It appears that QCD factorization predictions 78 for direct $C P$ violation seem not consistent with experiment, whereas pQCD results 9 are in the right ballpark. Recalling that sizable strong phases can be induced from the annihilation diagram in the pQCD approach by introducing the parton's transverse momentum, this means that we have to explore the FSI rescattering phases seriously in QCD factorization which are unlikely to be small possibly causing large compound $C P$-violating partial rate asymmetries 
in aforementioned charmless decay modes. The sizable $C P$ asymmetry observed in $\bar{B}^{0} \rightarrow K^{-} \pi^{+}$decays is a strong indication for large direct $C P$ violation driven by long-distance rescattering effects.

Besides the above-mentioned $C P$ violation, there exist some other hints at large FSI effects in the $B$ physics sector; see $\underline{6}$ for details.

\section{Final State Interactions in charmless $B$ decays}

Based on the Regge approach, Donoghue et al! $!^{10}$ have reached the interesting conclusion that FSIs do not disappear in the heavy quark limit and soft FSI phases are dominated by inelastic scattering, contrary to the common wisdom. A few years later, it was pointed out by Beneke et al. 7 within the framework of QCD factorization that the above conclusion holds only for individual rescattering amplitudes. When summing over all possible intermediate states, there exist systematic cancellations in the heavy quark limit so that the strong phases must vanish in the limit of $m_{b} \rightarrow \infty$. Hence, the FSI phase is generally of order $\mathcal{O}\left(\alpha_{s}, \Lambda_{\mathrm{QCD}} / m_{b}\right)$. In reality, because the $b$ quark mass is not very large and far from being infinity, the aforementioned cancellation may not occur or may not be very effective for the finite $B$ mass. Moreover, the strong phase arising from power corrections can be in principle very sizable. Therefore, we will model FSIs as rescattering processes of some intermediate two-body states with one particle exchange in the $t$-channel and compute the absorptive part via the optical theorem.

The calculations of hadronic diagrams for FSIs involve many theoretical uncertainties. Since the one particle exchange in the $t$ channel is off shell and since final state particles are hard, form factors or cutoffs must be introduced to the strong vertices to render the calculation meaningful in perturbation theory. As we do not have first-principles calculations for form factors, we shall use the measured rates to fix the unknown cutoff parameters and then use them to predict direct $C P$ violation. The results are shown in the last column of Table 1 . We see that direct $C P$-violating partial rate asymmetries in $K^{-} \pi^{+}, \rho^{+} \pi^{-}$and $\pi^{+} \pi^{-}$modes are significantly affected by final-state rescattering and their signs are different from that predicted by the short-distance approach.

It is worth stressing a subtle point for $B \rightarrow \pi \pi$ decays. The rescattering charming penguins in $\pi \pi$ are suppressed relative to that in $K \pi$ modes as the former are Cabibbo suppressed. Consequently, charming penguins are not adequate to explain the $\pi \pi$ data: the predicted $\pi^{+} \pi^{-}\left(\sim 9 \times 10^{-6}\right)$ is too large whereas $\pi^{0} \pi^{0}$ $\left(\sim 0.4 \times 10^{-6}\right)$ is too small. This means that a dispersive contribution is needed to interfere destructively with $\pi^{+} \pi^{-}$so that $\pi^{+} \pi^{-}$will be suppressed while $\pi^{0} \pi^{0}$ will get enhanced. This contribution cannot arise from the charming penguins or otherwise it will also contribute to $K \pi$ significantly and destroy all the nice predictions for $K \pi$. In the topological diagrammatic approach, 11 this dispersive term comes from the so-called vertical $W$-loop diagram $\mathcal{V}$ in which meson annihilation such as $D^{+} D^{-} \rightarrow \pi \pi$ occurs. 


\section{Polarization Anomaly in $B \rightarrow \phi K^{*}$}

For $B \rightarrow V_{1} V_{2}$ decays with $V$ being a light vector meson, it is expected that they are dominated by longitudinal polarization states and respect the scaling law: $1-f_{L}=$ $\mathcal{O}\left(m_{V}^{2} / m_{B}^{2}\right)$. However, a low value of the longitudinal fraction $f_{L} \approx 50 \%$ in $\phi K^{*}$ decays was observed by both BaBar 12 and Belle 13 . This polarization anomaly poses an interesting challenge for any theoretical interpretation.1415

Since the scaling law is valid only at short distances, one can try to circumvent it by considering the long-distance rescattering contributions from intermediate states $D^{(*)} D_{s}^{(*)}$. The large transverse polarization induced from $B \rightarrow D^{*} D_{s}^{*}$ will be propagated to $\phi K^{*}$ via FSI rescattering. Furthermore, rescattering from $B \rightarrow D^{*} D_{s}$ or $B \rightarrow D D_{s}^{*}$ will contribute only to the $A_{\perp}$ amplitude. Recently, we have studied FSI effects on $B \rightarrow V V$. While the longitudinal polarization fraction can be reduced significantly from short-distance predictions due to such FSI effects, no sizable perpendicular polarization is found owing mainly to the large cancellations occurring in the processes $B \rightarrow D_{s}^{*} D \rightarrow \phi K^{*}$ and $B \rightarrow D_{s} D^{*} \rightarrow \phi K^{*}$ and this can be understood as a consequence of $C P$ and $\mathrm{SU}(3)$ symmetry. Our result is different from a recent similar study in 15 . To fully account for the polarization anomaly (especially the perpendicular polarization) observed in $B \rightarrow \phi K^{*}$, FSI from other states or other mechanism, e.g. the penguin-induced annihilation, may have to be invoked.

The same FSI mechanism will also induce sizable transverse polarization in $B \rightarrow$ $\rho K^{*}$ decays. We found that $f_{L}\left(\rho K^{*}\right)$ is reduced to about $60 \%$ which is consistent with data for $\rho^{ \pm} K^{* 0}$ but not for $\rho^{0} K^{* \pm}$. This should be clarified experimentally.

\section{Acknowledgments}

I am grateful to Chun-Khiang Chua and Amarjit Soni for very fruitful collaboration.

\section{References}

1. BaBar Collaboration, B. Aubert et al., hep-ex/0407057

2. Belle Collaboration, Y. Chao et al., hep-ex/0408100

3. Heavy Flavor Averaging Group, http://www.slac.stanford.edu/xorg/hfag

4. Belle Collaboration, K. Abe et al., Phys. Rev. Lett. 93, 021601 (2004).

5. BaBar Collaboration, B. Aubert et al., hep-ex/0408089

6. H.Y. Cheng, C.K. Chua, and A. Soni, hep-ph/0409317

7. M. Beneke, G. Buchalla, M. Neubert, and C.T. Sachrajda, Phys. Rev. Lett. 83, 1914 (1999); Nucl. Phys. B 591, 313 (2000); ibid. 606, 245 (2001).

8. M. Beneke and M. Neubert, Nucl. Phys. B 675, 333 (2003).

9. Y.Y. Keum, H.n. Li, and A.I. Sanda, Phys. Rev. D 63, 054008 (2001); Y.Y. Keum and A.I. Sanda, ibid. 67, 054009 (2002).

10. J.F. Donoghue et al., Phys. Rev. Lett. 77, 2178 (1996).

11. L.L. Chau and H.Y. Cheng, Phys. Rev. Lett. 56, 1655 (1986).

12. BaBar Collaboration, B. Aubert et al., Phys. Rev. Lett. 91, 171802 (2003).

13. Belle Collaboration, K.F. Chen et al., Phys. Rev. Lett. 91, 201801 (2003).

14. A.L. Kagan, hep-ph/0405134 W.S. Hou and M. Nagashima, hep-ph/0408007

15. P. Colangelo, F. De Fazio, and T. N. Pham, Phys. Lett. B 597, 291 (2004). 